ISSN 1823-626X

Journal of Fundamental Sciences

available online at http://jfs.ibnusina.utm.my

\title{
P-SV wave diffusion in fluid-saturated medium
}

Dennis Ling C.C. and Zainal A.A.*

Department of Mathematics, Faculty of Science, Universiti Teknologi Malaysia, 81310 Skudai Johor, Malaysia.

Received 10 April 2010, Revised 19 June 2010, Accepted 21 June 2010, Available online 25 June 2010

\begin{abstract}
Propagating P-SV waves in the fluid-saturated mediums are categorized to fall into two distinct groups: insoluble and soluble mediums. These waves are known as surface Rayleigh waves. By introducing these waves with slowness in accordance to Snell Law, the diffusive and rotating waves are obtained. The results bear out that the propagating P-SV waves in soluble medium share similar diffusion characteristic as of insoluble medium while the discussions on fluid density in the mediums show that high density fluid promotes diffusive characteristic while low density fluid endorses nondiffusive P-SV waves. There is a compressed zone during the propagation of P-SV waves in medium saturated with high density fluids.
\end{abstract}

| Diffusion| Attenuation | Diffraction | Shock waves |

(R) 2010 Ibnu Sina Institute. All rights reserved. http://dx.doi.org/10.11113/mjfas.v6n1.178

\section{INTRODUCTION}

P-waves refer to the primary waves when an earthquake is discussed. Engineers estimated the wet-rock $\mathrm{P}$-wave velocity from the dry-rock P-wave velocity [1]. In geophysics, $\mathrm{S}$ waves are categorized to $\mathrm{SV}$ and $\mathrm{SH}$ waves while the combination of P-SV waves are known as surface Rayleigh waves in [10]. The dependence of P- and S-wave attenuation on strain amplitude and frequency had been studied experimentally in dry and water-saturated sandstone samples under a confining pressure [2]. The protection of underground structures against dynamic loadings has long been a topic of great interest in defense engineering. Hence, the P-wave propagation and attenuation in rock shelter layer with an inclusion or filled medium had been studied [3]. The problem of diffraction of waves due to plane harmonic P-wave incident normally on a line crack situated in an infinite micro-polar elastic medium was studied as well in [4]. Nevertheless, an analytical solution is obtained for twodimensional scattering and diffraction of plane $\mathrm{P}$ waves by circular-arc alluvial valleys with shallow saturated soil deposits [12]. In Cardiology, P-waves had been used for monitoring in risk assessment of the patient after surgery [13].

The dependence of $\mathrm{P}$ - and S-wave attenuation to strain amplitude and frequency in saturated medium is studied in [2]. Investigation on saturation on horizontal and vertical of porous soils was carried out in [14].

Corresponding author at: Department of Mathematics, Faculty of Science

Universiti Teknologi Malaysia, 81310 Skudai Johor, Malaysia.

E-mail addresses: zainalaz@utm.my (Zainal A.A.)
As the wave's amplitude increases, the frequency increases too. Effects of saturation on the velocities of $\mathrm{P}$ - and $\mathrm{S}$ waves in several types of soil are studied and the outcomes show that the velocity is higher in dense sand. Similar outcome is found in [1]. The thickness of the porous medium is related to the frequency of the waves. The frequency decreases when the thickness of the medium increases [7]

Hence, this study aims to probe the connection of PSV wave's refraction velocity and the attenuation. The derivation of P-SV waves will be shown by the analytic discussion. Some figures will be plotted to give better viewing pleasure.

\section{EXPERIMENTAL}

The governing equations:

$\mu \nabla^{2} u+(\lambda+\mu) \nabla(\nabla u)+\rho F=\rho \frac{\partial^{2} u}{\partial t^{2}}$

Boundary conditions for $z=0$ :

$\sigma_{z} \equiv(\lambda+\mu) \frac{\partial u_{z}}{\partial z}+\lambda\left(\frac{\partial u_{x}}{\partial x}+\frac{\partial u_{y}}{\partial y}\right)=0$

$\tau_{x z} \equiv \mu\left(\frac{\partial u_{x}}{\partial z}+\frac{\partial u_{z}}{\partial x}\right)=0$

$\tau_{y z} \equiv \mu\left(\frac{\partial u_{y}}{\partial z}+\frac{\partial u_{z}}{\partial y}\right)=0$

Initial conditions for $t=0$ : 


$$
\begin{aligned}
& u(x, y, z)=0 \\
& \frac{\partial u_{x}}{\partial t}=\frac{\partial u_{y}}{\partial t}=\frac{\partial u_{z}}{\partial t}=0
\end{aligned}
$$

In linear elasticity for isotropic medium, $\lambda$ and $\mu$ denote the Lame parameters for the stress $\sigma_{\mathrm{z}}, \tau_{\mathrm{xz}}, \tau_{\mathrm{yz}}$ and the displacements $u_{x}, u_{y}$ and $u_{z}$ are continuous everywhere.

$F$ is the body force in the direction of $x, y, z$ respectively and $\rho$ is the density.

Provided that $\omega$ is the angular velocity or frequency, $k$ is the wave number, $c$ is the wave velocity along with the dispersion relation $\omega=c k$, the group velocity of a wave is the velocity with the overall shape of the wave's amplitudes which is also known as envelope of the waves. In other words, the phase velocity is the average velocity of the components, given by $V_{p}=\omega / k$. The group velocity is velocity of the envelope, given by $V_{g}=d \omega / d k$ [9].

When this is applied to the problem of P-SV wave's propagation in fluid saturated medium, the group velocity reflects the apparent velocity of the surface displacement or the overall shape of the P-SV wave's amplitude at the boundary of the medium. The envelope is formed by the phase velocity of P-SV waves. In this research, the apparent velocity or surface displacement velocity is measured along the boundary of the similar density medium in accordance to Snell law:

$$
\frac{V_{a p p}}{\sin f}=\frac{c}{\sin e} \text {, }
$$

where $e$ is the incident angle and $f$ is the refraction angle made by the P-SV waves. However, this research aims to study P-SV waves in horizontal direction only. For the case of fluid-saturated medium, there exists variation in density within medium [7, 8]. There exists slowness for fluidsaturated medium $[5,6]$ i.e. the apparent velocity of the displacement at the boundary is slower than phase velocity of the wave in the medium that gives

$V_{a p p}<c$.

In this study, the elastic wave equation will be solved for extracting P-SV wave's displacements in the different types of medium that satisfied (5) and (6). By using the divergence operator

$$
\nabla^{2} u=\nabla(\nabla u)-\nabla \times(\nabla \times u),
$$

eq. (1) is reduced to

$$
\begin{aligned}
& \alpha^{2} \nabla(\nabla \cdot u)-\beta^{2} \nabla \times \nabla \times u+F=\frac{\partial^{2} u}{\partial t^{2}}, \\
& \alpha=\sqrt{\frac{\lambda+2 \mu}{\rho}}, \beta=\sqrt{\frac{\mu}{\rho}},
\end{aligned}
$$

where $\alpha$ and $\beta$ are the velocities for the P-wave and $\mathrm{S}$ wave and Eq. (8) is the elastic wave equation [10]. Hence, this modelling is only valid for elastic medium and it is necessary to reduce the right hand side (RHS) of (8) by letting

$u=\exp [i(k x-c t)]$.

By inserting second order derivative of (10) into RHS of (8), the equation is given by

$\alpha^{2} \nabla(\nabla \cdot u)-\beta^{2} \nabla \times \nabla \times u+F=-\omega^{2} u$.

Since elastic wave consists of irrotational $\mathrm{P}$-wave and solenoidal by S-wave [10], the displacement shall be written as

$u=u_{P}+u_{S}$.

For irrotational P-waves, the vorticity $\nabla \times u_{p}=0$. Thus, the relation (12) becomes

$\nabla u_{p} \neq 0, \nabla \times u_{p}=0, \nabla u_{S}=0, \nabla \times u_{P} \neq 0$,

By inserting (12) and (13) into (11), the equation yields

$\alpha^{2}\left(\nabla^{2} u_{P}+k_{P}^{2} u_{p}\right)+\beta^{2}\left(\nabla^{2} u_{s}+k_{s}^{2} u_{s}\right)=0$,

$\omega=\frac{\alpha}{k_{p}}, \frac{\beta}{k_{s}}, F=0$.

The Helmholtz equations [11] for P- and S-waves are given by

$\nabla^{2} u_{P}+k_{P}^{2} u_{P}=0, \nabla^{2} u_{s}+k_{s}^{2} u_{s}=0$.

Here, the Helmholtz equations are solved by utilizing Hansen vector [10] that gives

$u_{p}=A\left(l a_{x}+n a_{z}\right) \exp \left[i \omega\left(t-\frac{l x+n z}{\alpha}\right)\right]$,

$u_{S V}=B\left(-n a_{x}+l a_{z}\right) \exp \left[i \omega\left(t-\frac{l x+n z}{\beta}\right)\right]$,

$u_{S H}=C a_{y} \exp \left[i \omega\left(t-\frac{l x+n z}{\beta}\right)\right]$,

where $a_{x}, a_{y}$ and $a_{z}$ are the unit vectors while $l$ and $n$ are the vector components. Eqs. (17) and (18) will be considered in this study since the aim is to study the P-SV waves only.

P-SV wave's incidence is illustrated in Fig. 1. The amended eqs. (17) and (18) in which the amplitudes reduce with depth are

$u_{P}=A\left(a_{x}+\eta_{\alpha} a_{z}\right) \exp \left[i k\left(c t-x-\eta_{\alpha} z\right)\right]$,

$\eta_{\alpha}=\sqrt{\frac{c^{2}}{\alpha^{2}}-1}$,

$u_{S V}=B\left(\eta_{\beta} a_{x}+a_{z}\right) \exp \left[i k\left(c t-x-\eta_{\beta} z\right)\right]$,

$\eta_{\beta}=\sqrt{\frac{c^{2}}{\beta^{2}}-1}$,

where $\eta_{\alpha}$ and $\eta_{\beta}$ are always positive. The velocity of the PSV waves, $\alpha$ and $\beta$ are measured at the boundary of the 
medium or $z=0$ and these are similar to the apparent velocity $V_{\text {app }}$ of the surface displacement. For the displacement normal to the propagation direction by means of quantity $\eta_{\alpha}$ and $\eta_{\beta}$ with amplitudes $A$ and $B$, eqs. (20a) and (20b) for $x$-direction of displacements with amplitude reduces with depth $z$ gives

$u_{P-S V}=\left[A\left(a_{x}+\eta_{\alpha} a_{z}\right) \exp \left(i k \eta_{\alpha} z\right)+B\left(\eta_{\beta} a_{x}+a_{z}\right) \exp \left(i k \eta_{\beta} z\right)\right] \exp [i k(a-x)]$

, $\omega_{1}=\eta_{\alpha} k$ or $\eta_{\beta} k$.

Here, the quantities $\eta_{\alpha}$ and $\eta_{\beta}$ refer to the refracted wave velocities that give $\omega_{1}=\eta_{\alpha} k$ or $\eta_{\beta} k$ while the vector $a_{z}$ shows the displacement is at $z$-direction while the P-SV waves propagate in $x$-direction. From wave terminology, the term $A\left(a_{x}+\eta_{\alpha} a_{z}\right) \exp \left(i k \eta_{\alpha} z\right)+B\left(\eta_{\beta} a_{x}+a_{z}\right) \exp \left(i k \eta_{\beta} z\right)$ in eq. (21) will show the plane waves that are diffusive with depth $z$ of $\omega_{1}=\eta_{\alpha} k$ or $\eta_{\beta} k$ is in complex [9]. The diffusive waves are associated with attenuation of the amplitudes with the time due to certain dissipation mechanisms present in the system. Eq. (21) should be polarized to give the real quantities (frequency) equation that reads [10].

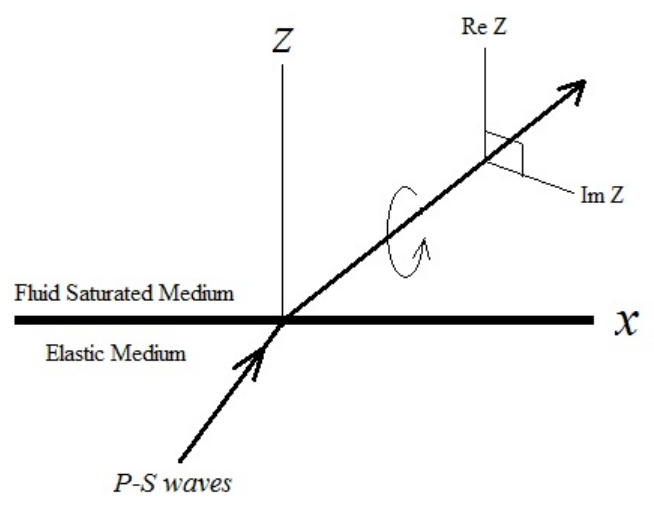

Figure 1: The P-SV wave's incidence and the fluid-saturated medium.

$$
u_{p-S V}=\left[A \eta_{\alpha} \exp \left(i k \eta_{\alpha} z\right)+B\left(1 / \eta_{\beta}\right) \exp \left(i k \eta_{\beta} z\right)\right] \exp [i k(c t-x)]
$$

Next, the roles of $\eta_{\alpha}$ and $\eta_{\beta}$ will be shown. For the fluidsaturated medium, the slowness is induced by refraction [5, 6]. The envelope velocity at medium surface is different with wave velocity in the medium after the slowness or $V_{a p p} \neq c$. For particular case, the P-SV waves' velocity in the medium is greater than the envelope's velocity that yields

$c>\alpha, c>\beta, V_{\text {app }}=\alpha, \beta$.

Here, we propose the relations for another two types of medium conditions such that

$$
\begin{aligned}
& c=\alpha, c=\beta, V_{a p p}=\alpha, \beta, \\
& c<\alpha, c<\beta, V_{\text {app }}=\alpha, \beta .
\end{aligned}
$$

We note that the relations (23) and (25) are meant for the insoluble medium such that the variation of velocities $c$ and $\alpha$ or $\beta$ is significant. When the velocity $c$ is similar to $\alpha$ or $\beta$, we presume this will explain the soluble medium such that the fluid mixes well with the medium to give similar velocity.

Yang and Tadanobu [14] and Kahraman [1] show that the high density medium promotes high wave's velocity. Hence, the relation (23) will only be present when the low density fluid is saturated in the insoluble medium; the low density fluid will reshuffle the ray velocity or reduce the P-SV wave velocity. Eventually, the relation (25) is meant for the high density fluid saturated in the insoluble medium.

The detailed explanations about the tie between medium's solubility and the fluid's density will be discussed next for showing the vital roles play by the relations (23-25) especially the attenuation's characteristic. When condition (25) is applied to the Eq. (21), a complex solution will be obtained for $\eta_{\alpha}$ and $\eta_{\beta}$ that reads

$\eta_{\alpha}=i \eta_{\alpha}, \eta_{\beta}=i \eta_{\beta}$

This indicates a modification is required for Eq. (22) to give (27). Hence, the P-SV wave's displacements for 3 types of mediums are

$u_{P-S V}=\left[A \eta_{\alpha} \exp \left(k \eta_{\alpha} z\right)+B\left(1 / \eta_{\beta}\right) \exp \left(k \eta_{\beta} z\right)\right] \exp [i k(c t-x)]$

for $c<\alpha, c<\beta$,

$u_{p-S V}=\exp [i k(c t-x)]$ for $c=\alpha, c=\beta$,

$u_{P-S V}=\left[A \eta_{\alpha} \exp \left(i k \eta_{\alpha} z\right)+B\left(1 / \eta_{\beta}\right) \exp \left(i k \eta_{\beta} z\right)\right] \exp [i k(c t-x)]$

for $c>\alpha, c>\beta$,

Eq. (27-29) show that the P-SV wave's propagation in $x$ direction whiles the diffusion is in z-direction. The Eq. (2729) are arranged such that the density of the saturated fluid reduces from $c<\alpha, \beta$ to $c>\alpha, \beta$. Figs. 2 and 3 are plotted respectively.

\section{RESULTS}

Fig. 2 is plotted for the diffusive P-SV waves with $c<\alpha, c<\beta \quad-5 \leq x \leq 5, k=1, \alpha=4, \beta=4 c=2$. High density fluid promotes the diffusive characteristic for propagating P-SV waves in fluid saturated medium. The amplitude diminishes at medium surface or $z=0$ as shown in fig. 2(b). Fig. 2(a) illustrates the rotating behaviour of P-SV waves. There is a compression zone by P-SV wave's rotation which is known as shock waves [15].

Insoluble medium with low density fluid (29) is nondiffusive given that the term $\omega_{1}=\eta_{\alpha} k$ or $\eta_{\beta} k$ is in real [9].

Fig. 3 is plotted for the non-diffusive P-SV waves with $c>\alpha, c>\beta,-5 \leq x \leq 5, k=1, \alpha=2, \beta=2, c=4$. It is 
shown in Fig. 3(a) the P-SV waves are polarized evenly in $x-Z$ direction. The compression which is related to shock wave is formed by the wave diffraction at the medium surface [15].

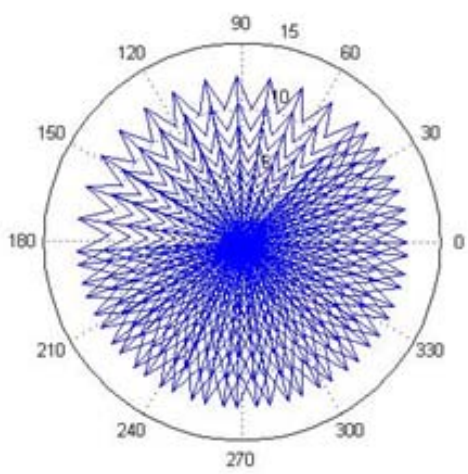

(a)
Fig 3(b) illustrates the non diffusive characteristic of P-SV waves. The P-SV waves are non diffusive since the celerity of the P-SV waves are constant.



(b)

Figure 2: The P-SV wave's propagates in the medium saturated with high density fluid, $c<\alpha, c<\beta-5 \leq x \leq 5, k=1, \alpha=4$ $\beta=4, c=2$. (a) The rotating behaviour of P-SV waves. (b) The diffusive characteristic of P-SV waves.

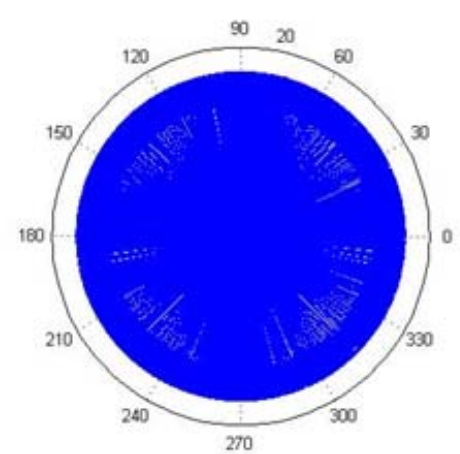

(a)

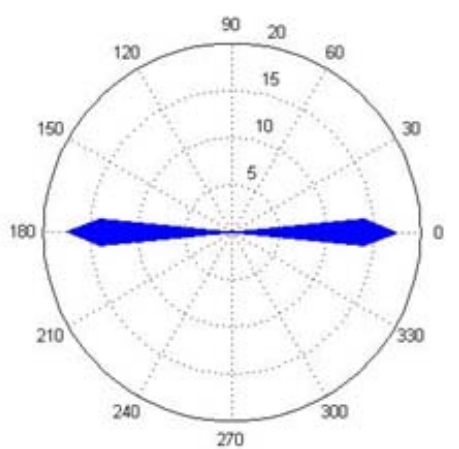

(b)

Figure 3: The P-SV wave's propagates in the medium saturated with low density fluid, $c>\alpha, c>\beta,-5 \leq x \leq 5, k=1, \alpha=2, \beta=2$, $c=4$. (a) The rotating behaviour of P-SV waves. (b) The non-diffusive characteristic of P-SV waves.

\section{DISCUSSIONS}

For soluble medium, Eq. (28) gives

$u_{p-S V}=\exp [i k(c t-x)], \eta_{\alpha}, \eta_{\beta}=0$

Relation (30) implies that the P-SV wave in soluble medium is non-diffusive plane wave and there is no diffraction. Insoluble medium with displacement (27) is diffusive given that the term $\omega_{1}=\eta_{\alpha} k$ or $\eta_{\beta} k$ is complex in accordance to (26). Apparently, the shock wave is illustrated in medium saturated with both high and low density fluid in accordance to Figs. 2 and 3. The compressed zone of waves in figure 2(a) shows that there is possibility of generating significant displacement during an earthquake. The existence of lower density fluid is disastrous since the waves are not diffusive. The amplitude is not reduced immediately for which the displacement remains until the higher density fluid or soluble medium exists.

\section{CONCLUSION}

The studies show that the P-SV waves in soluble medium are non-diffusive. However, the discussion on fluid density has linked the P-SV waves to the diffusion. High density fluid in insoluble medium promotes diffusive P-SV waves while low density fluid in insoluble medium promotes non-diffusive P-SV waves. The non-diffusive waves have induced shock wave in the insoluble medium after diffraction. Parts of the rotating P-SV waves are compressed during the propagation of shock waves in the medium saturated with higher density. However, there are less compressed waves for which the shock waves are assumed less disastrous to the medium.

\section{ACKNOWLEDGEMENT}

DLCC is indebted to MOSTI for NSF. This research is partially funded by MOHE FRGS Vote no 78485. 


\section{REFERENCES}

[1] S. Kahraman, Ultrasonics 46 (2007) 341-348. doi:10.1016/j.ultras.2007.05.003 .

[2] E.I. Mashinskii, Russian Geology and Geophysics 50 (2009) 734-738. doi:10.1016/j.rgg.2008.12.013

[3] Z.L. Wang, H.Konietzky, R.F.Shen, Soil Dynamics and Earthquake Engineering 30 (2010) 1-7 doi:10.1016/j.soildyn.2009.05.004.

[4] G.K. Midya, G.C. Layek, T.K. De, International Journal of Solids and Structures 45 (2008) 2706-2722. doi:10.1016/j.ijsolstr.2007.12.020

[5] C.M. Keith, S. Crampin, Geophys. J. R. Astr. Soc., 49(1977) 181-208. doi: 10.1111/j.1365-246X.1977.tb03708.x

[6] M.D. Sharma, J. Earth Syst. Sci., 116(2007) 537-551. http://www.springerlink.com/content/u48511327r02893x/fulltext.pdf

[7] J.T. Wang, C.H. Zhang and F. Jin. Int. J. Numer. Anal. Methods Geomech., 33(2009) 1467-1484. doi: 10.1002/nag.773.

[8] M.D. Sharma, Geophysical Journal International (2004), doi:10.1111/j1365-246x.2004.02226.x.

[9] P.L. Bhatnagar, Nonlinear Waves in One-dimensional Dispersive System: edition 1. Thomson Press, India, 1977 pp: 4-20. ISBN: 0-19-853531-7

[10] J.A. Pujol, Elastic Wave Propagation and Generation in Seismology: edition 1. Cambridge University Press, United Kingdom, 2003 pp: $124-125$. ISBN: 0-521-52046-1

[11] B. Gang, G.W. Wei and Z. Shan, Int. J. Numer. Meth. Eng., 59(2004) 389-408. doi: 10.1002/nme.883

[12] L. Weihua, Z. Chenggang, S. Peixin, Soil Dynamics and Earthquake Engineering, 25 (2005) 997-1014 doi:10.1016/j.soildyn.2004.10.010

[13] C. Materazzo, P. Piotti, C. Mantovani, R. Miceli, F. Villani, European Journal of Cardio-thoracic Surgery, 31 (2007) 812-816. doi:10.1016/j.ejcts.2007.02.007

[14] J. Yang, S. Tadanobu, Soil Dyn. Earthquake Eng., 19(2000) 339-346. doi: 10.1016/S0267-7261(00)00023-3

[15] Z. Han, and X. Yin, Shock Dynamics: edition 1. Kluwer Academic Publisher, Chin, 1993 pp: 42-48. ISBN:0-7923-1746-7. 